(C) 2016 IEEE. Personal use of this material is permitted. Permission from IEEE must be obtained for all other uses, in any current or future media, including reprinting/republishing this material for advertising or promotional purposes, creating new collective works, for resale or redistribution to servers or lists, or reuse of any copyrighted component of this work in other works. 


\title{
Uplink Performance Analysis of Dense Cellular Networks with LoS and NLoS Transmissions
}

\author{
Tian Ding ${ }^{\dagger}$, Ming Ding ${ }^{\ddagger}$, Guoqiang Mao ${ }^{\dagger \star * \S}$, Zihuai Lin ${ }^{\star}$, David López-Pérez \\ †School of Computing and Communications, The University of Technology Sydney, Australia \\ ${ }^{\ddagger}$ Data61, Australia \\ * School of Information and Communication Engineering, Beijing University of Posts and Telecommunications, Beijing, China

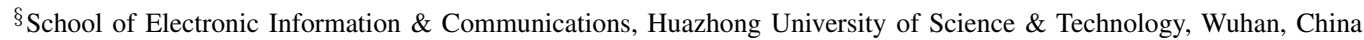 \\ ${ }^{\star}$ The University of Sydney, Australia \\ ๑Bell Labs Alcatel-Lucent, Ireland
}

\begin{abstract}
In this paper, we analyse the coverage probability and the area spectral efficiency (ASE) in the uplink (UL) of dense small cell networks (SCNs) considering a piecewise linear path loss model incorporating both line-of-sight $(\mathrm{LOS})$ and non-lineof-sight (NLoS) transmissions. The performance impact of LoS and NLoS transmissions in the ASE of the UL of dense SCNs is shown to be significant, both quantitatively and qualitatively, compared with previous works that do not differentiate LoS and NLoS transmissions. In particular, previous works predicted that a larger UL power compensation factor would always result in a better ASE in the practical range of user equipment (UE) density, i.e., $10^{0} \sim 10^{3} \mathbf{U E s} / \mathbf{k m}^{2}$. However, our results show that a smaller UL power compensation factor can greatly boost the ASE in the UL of dense SCNs, i.e., $10^{2} \sim 10^{3} \mathbf{U E s} / \mathbf{k m}^{2}$, while a larger UL power compensation factor is more suitable for sparse SCNs, i.e., $10^{0} \sim 10^{2} \mathbf{U E s} / \mathbf{k m}^{2}$.
\end{abstract}

\section{INTRODUCTION}

Small cell networks (SCNs) can achieve a high spatial reuse through network densification, which in turn can significantly enhance network capacity $[1,2]$. Particularly, the orthogonal deployment of SCNs within the existing macrocell network, i.e., small cells and macrocells operating on different frequency spectrum (Small Cell Scenario \#2a defined in [3]), was prioritized in the design of the 4th generation (4G) Long Term Evolution (LTE) networks by the 3rd Generation Partnership Project (3GPP), and dense SCNs are envisaged to be the workhorse for capacity enhancement in the 5th generation (5G) networks due to its large performance gains and its easy deployment [1]. Thus, this paper focuses on studying the performance of these orthogonal deployments of dense SCNs.

In order to deploy dense SCNs in a cost-effective manner, a deep theoretical understanding of the implications that small cells bring about is needed. Using stochastic geometry theory, the authors of [4] and [5] respectively conducted network performance analyses for the downlink (DL) and the uplink (UL) of SCNs, assuming that user equipments (UEs) and/or base stations (BSs) are randomly deployed according to a homogeneous Poisson distribution. In [6], the authors considered UE spatial blocking, which is referred to the outage caused by limited number of usable channels, and derived approximate expressions for the UL blocking probability and the UL coverage probability. In [7], the authors proposed a tractable model to characterize the UL rate distribution in a $K$-tier heterogeneous cellular networks (HCNs) considering power control and load balancing. In [8], the authors considered the maximum power limitation for UEs and obtained approximate expressions for the UL outage probability and UL spectral efficiency. However, none of these UL related works considered a realistic path loss model with line-of-sight (LoS) and non-line-of-sight (NLoS) transmissions.

In our previous work [9], we conducted a study on the DL of dense SCNs considering a sophisticated path loss model that differentiates LoS and NLoS transmissions. LoS transmission may occur when the distance between a transmitter and a receiver is small, and NLoS transmission is more common in office environments and in central business districts. Moreover, the probability that there exists a LoS path between the transmitter and receiver increases as the distance decreases. The decrease of the distance between the transmitter and receiver will cause a transition from NLoS transmission to LoS transmission with a higher probability.

In this paper, we extend our previous work [9] to study the performance impact of such NLoS-to-LoS transition in the UL of dense SCNs. The main contributions of this paper are as follows:

- Numerically tractable results are obtained for the UL coverage probability and the UL area spectral efficiency (ASE) performance using a piecewise path loss model, incorporating both $\mathrm{LoS}$ and NLoS transmissions. To evaluate the numerical results and calcualte integrals efficiently, we apply the Gauss-Laguerre quadrature [11].

- Our theoretical analysis of the UL of dense SCNs shows a similar performance trend that was found for the DL of dense SCNs in our previous work [9], i.e., when the density of UEs is larger than a threshold, the ASE may suffer from a slow growth or even a decrease. Then, the ASE will grow almost linearly as the UE density increases above another larger threshold. This finding is in stark contrast with previous results using a simplistic path loss model that does not differentiate LoS and NLoS transmissions [5]. 
- Our theoretical analysis also indicates that the performance impact of LoS and NLoS transmissions on the UL of SCNs with UL power compensation is significant both quantitatively and qualitatively with respect to existing works in the literature. In particular, the previous work [5] showed that a larger UL power compensation factor should always deliver better ASE performance in the practical range of UE density, i.e., $10^{0} \sim 10^{3} \mathrm{UEs} / \mathrm{km}^{2}$. However, our results show that a smaller UL power compensation factor can greatly boost the ASE performance in dense SCNs, i.e., $10^{2} \sim 10^{3} \mathrm{UEs} / \mathrm{km}^{2}$, while a larger UL power compensation factor is more suitable for sparse SCNs, i.e., $10^{0} \sim 10^{2} \mathrm{UEs} / \mathrm{km}^{2}$. Our new finding indicates that it is possible to save UE battery and meanwhile obtain high ASE in the UL of dense SCNs in $5 \mathrm{G}$, if the UL power compensation factor is optimized.

\section{SYSTEM MODEL}

In this paper, UEs are distributed following a homogeneous Poisson point process (HPPP) with density $\lambda \mathrm{UEs} / \mathrm{km}^{2}$, and associate with its nearest BS, thus creating Voronoi cells. The distance from the typical BS located at the origin to the typical UE is denoted as $R$, where $R$ is a random variable i.i.d. Rayleigh distributed [5] and its probability density function (PDF) can be calculated as

$$
f_{R}(r)=2 \pi \lambda R \times \exp \left(-\lambda \pi R^{2}\right) .
$$

The link from the typical BS to the typical UE has a LoS link or a NLoS link with probability $\operatorname{Pr}^{\mathrm{L}}(R)$ and $1-\operatorname{Pr}^{\mathrm{L}}(R)$, respectively, where such probability can be computed by the following linear function $[9,10]$

$$
\operatorname{Pr}^{\mathrm{L}}(R)=\left\{\begin{array}{ll}
1-\frac{R}{d_{1}}, & 0<R \leq d_{1} \\
0, & R>d_{1}
\end{array},\right.
$$

where $d_{1}$ is the cut-off distance of the LoS link.

The distance dependent path loss is expressed as $R^{-\alpha}$, where the path loss exponent of each link is

$$
\alpha=\left\{\begin{array}{ll}
\alpha^{\mathrm{L}}, & \text { LoS with probability } \operatorname{Pr}^{\mathrm{L}}(R) \\
\alpha^{\mathrm{NL}}, & \text { NLoS with probability }\left(1-\operatorname{Pr}^{\mathrm{L}}(R)\right)
\end{array} .\right.
$$

The typical UE transmits with a baseline power $P_{0} \mathrm{~mW}$, and uses a distance-based fractional power compensation of $R^{\alpha \epsilon}$ [12], where $\epsilon$ is the UL power compensation factor.

Therefore, the received signal power at the typical BS can be written as

$$
P^{\mathrm{sig}}=P_{0} g R^{\alpha(\epsilon-1)},
$$

where $g$ denotes the channel gain of the multi-path fading and is a random variable i.i.d. Rayleigh distributed [5]. Hence, $g$ follows an exponential distribution with unitary mean.

As a result, the SINR at the typical BS of the typical UE can be expressed as

$$
S I N R=\frac{P_{0} g R^{\alpha(\epsilon-1)}}{\sigma^{2}+I_{Z}}
$$

where $\sigma^{2}$ is the noise power, $Z$ is the set of interfering UEs, and $I_{Z}$ is the interference given by

$$
I_{Z}=\sum_{Z} P_{0}\left(R_{z}^{\alpha}\right)^{\epsilon} g_{z} D_{z}^{-\alpha}
$$

where the distance of interferer $z \in Z$ to the typical BS is denoted by $D_{z}$, and the distance of interferer $z \in Z$ to its serving $\mathrm{BS}$ is denoted by $R_{z}$. According to [5], $R_{z}$ is also i.i.d. Rayleigh distributed and its PDF can be calculated as (1).

\section{Analysis of Coverage Probability And ASE}

According to [5], the coverage probability can be formulated as

$$
P^{\mathrm{cov}}(\lambda, T)=\operatorname{Pr}[S I N R>T],
$$

where $T$ is the SINR threshold.

Based on the system model presented in Section II, we calculate $P^{\mathrm{cov}}(\lambda, T)$ and present it in the following lemma.

Lemma 1. $P^{c o v}(\lambda, T)$ can be derived as

$$
P^{c o v}(\lambda, T) \triangleq T_{1}^{\mathrm{L}}+T_{1}^{\mathrm{NL}}+T_{2}^{\mathrm{NL}}
$$

where

$$
\begin{aligned}
& T_{1}^{\mathrm{L}}=\int_{0}^{d_{1}} \operatorname{Pr}\left[\frac{P_{0} g r^{\alpha^{L}(\epsilon-1)}}{\sigma^{2}+I_{Z}}>T\right] \times\left(1-\frac{r}{d_{1}}\right) \times f_{R}(r) d r, \\
& T_{1}^{\mathrm{NL}}=\int_{0}^{d_{1}} \operatorname{Pr}\left[\frac{P_{0} g r^{\alpha^{N L}(\epsilon-1)}}{\sigma^{2}+I_{Z}}>T\right] \times\left(\frac{r}{d_{1}}\right) \times f_{R}(r) d r, \\
& T_{2}^{\mathrm{NL}}=\int_{d_{1}}^{\infty} \operatorname{Pr}\left[\frac{P_{0} g r^{\alpha^{N L}(\epsilon-1)}}{\sigma^{2}+I_{Z}}>T\right] f_{R}(r) d r .
\end{aligned}
$$

Proof: Given the distance distribution and the piecewise path loss model presente in Section II, $P^{\text {cov }}(\lambda, T)$ can be derived as

$$
\begin{aligned}
& P^{\operatorname{cov}}(\lambda, T) \\
& =\int_{0}^{\infty} \operatorname{Pr}[S I N R>T \mid R=r] f_{R}(r) d r \\
& =\int_{0}^{\infty} \operatorname{Pr}\left[\frac{P_{0} g r^{\alpha(\epsilon-1)}}{\sigma^{2}+I_{Z}}>T\right] f_{R}(r) d r \\
& =\int_{0}^{d_{1}} \operatorname{Pr}\left[\frac{P_{0} g r^{\alpha(\epsilon-1)}}{\sigma^{2}+I_{Z}}>T \mid \operatorname{LoS}\right] \times \operatorname{Pr}^{\mathrm{L}}(r) \times f_{R}(r) d r \\
& \quad+\int_{0}^{d_{1}} \operatorname{Pr}\left[\frac{P_{0} g r^{\alpha(\epsilon-1)}}{\sigma^{2}+I_{Z}}>T \mid \mathrm{NLoS}\right] \times\left(1-\operatorname{Pr}^{\mathrm{L}}(r)\right) \times f_{R}(r) d r \\
& \quad+\int_{d_{1}}^{\infty} \operatorname{Pr}\left[\frac{P_{0} g r^{\alpha(\epsilon-1)}}{\sigma^{2}+I_{Z}}>T \mid \mathrm{NLoS}\right] \times\left(1-\operatorname{Pr}^{\mathrm{L}}(r)\right) \times f_{R}(r) d r .
\end{aligned}
$$

Our proof is completed by plugging (3) and (2) into (9), and applying the definition of $T_{1}^{\mathrm{L}}, T_{1}^{\mathrm{NL}}$ and $T_{2}^{\mathrm{NL}}$ in (8).

Note that $T_{1}^{\mathrm{L}}, T_{1}^{\mathrm{NL}}$ and $T_{2}^{\mathrm{NL}}$ represent the coverage probability when the typical UE is associated with the typical BS using a LoS link of distance less than $d_{1}$, the coverage probability when the typical UE is associated with the typical BS using a a NLoS of distance less than $d_{1}$, and the coverage probability when the typical UE is associated with the typical BS using a NLoS of distance greater than $d_{1}$, respectively.

According to [9], the area spectral efficiency (ASE) in $\mathrm{bps} / \mathrm{Hz} / \mathrm{km}^{2}$ for a given $\lambda$ can be formulated as

$$
A^{\mathrm{ASE}}\left(\lambda, T_{0}\right)=\lambda \int_{T_{0}}^{\infty} \log _{2}(1+x) f_{X}(\lambda, x) d x
$$


where $T_{0}$ is the minimum working SINR for the considered SCN, and $f_{X}(\lambda, x)$ is the PDF of the SINR observed at the typical BS for a particular value of $\lambda$.

Based on the definition of $P^{\text {cov }}(\lambda, T)$, which is the complementary cumulative distribution function (CCDF) of SINR, $f_{X}(\lambda, x)$ can be computed as

$$
f_{X}(\lambda, x)=\frac{\partial\left(1-P^{\mathrm{cov}}(\lambda, x)\right)}{\partial x} .
$$

In the following subsections, we will investigate the results of $T_{1}^{\mathrm{L}}, T_{1}^{\mathrm{NL}}$ and $T_{2}^{\mathrm{NL}}$, respectively.

\section{A. The Result of $T_{1}^{\mathrm{L}}$}

Regarding the result of $T_{1}^{\mathrm{L}}$, which is the coverage probability when the typical UE is associated with the typical BS with a LoS link of distance less than $d_{1}$, we propose Lemma 2 in the following.

Lemma 2. When the typical UE is associated with a LoS BS of a distance less than $d_{1}$, the coverage probability $T_{1}^{\mathrm{L}}$ can be computed by

$T_{1}^{\mathrm{L}}=\int_{0}^{d_{1}} e^{-\frac{T \sigma^{2}}{P_{0} r^{\alpha^{L}(\epsilon-1)}}} \times \mathscr{L}_{I_{Z}}\left(\frac{T}{P_{0} r^{\alpha^{L}(\epsilon-1)}}\right) \times\left(1-\frac{r}{d_{1}}\right) \times f_{R}(r) d r$,

where the Laplace transform $\mathscr{L}_{I_{Z}}(s)$ is expressed as $\mathscr{L}_{I_{Z}}(s)=$

$\exp \left\{-2 \pi \lambda \int_{r}^{d_{1}}\left(1-\frac{x}{d_{1}}\right)\left(\mathbb{E}_{R_{z}, \alpha^{R_{z}}}\left[\frac{1}{1+\left(P_{0} s R_{z}^{R^{2} \epsilon} x^{-\alpha}\right)^{-1}}\right]\right) x d x\right\}$

$\times \exp \left\{-2 \pi \lambda \int_{r}^{d_{1}}\left(\frac{x}{d_{1}}\right)\left(\mathbb{E}_{R_{z}, \alpha R_{z}}\left[\frac{1}{1+\left(P_{0} s R_{z}^{\alpha} R_{z} \epsilon^{-\alpha^{N L}}\right)^{-1}}\right]\right) x d x\right\}$

$\times \exp \left\{-2 \pi \lambda \int_{d_{1}}^{\infty}\left(\mathbb{E}_{R_{z}, \alpha} R_{z}\left[\frac{1}{\left.1+\left(P_{0} s R_{z}^{\alpha}{ }^{R z} \epsilon_{x^{-\alpha}}\right)^{N L}\right)^{-1}}\right]\right) x d x\right\}$,

and the expectation function over $R_{z}$ and $\alpha^{R_{z}}$ is given by

$\mathbb{E}_{R_{z}, \alpha^{R z}}\left[\frac{1}{1+\left(P_{0} s R_{z}^{\alpha_{z} \epsilon_{x}-\alpha}\right)^{-1}}\right]$

$=\int_{0}^{d_{1}}\left(1-\frac{u}{d_{1}}\right) \frac{1}{1+\left(P_{0} s u^{\alpha^{L} \epsilon} x^{-\alpha}\right)^{-1}} 2 \pi \lambda u e^{-\pi \lambda u^{2}} d u$

$+\int_{0}^{d_{1}} \frac{u}{d_{1}} \frac{1}{1+\left(P_{0} s u^{\alpha^{N L}} \epsilon x^{-\alpha}\right)^{-1}} 2 \pi \lambda u e^{-\pi \lambda u^{2}} d u$

$+\int_{d_{1}}^{\infty} \frac{1}{1+\left(P_{0} s u^{\alpha^{N L} \epsilon} x^{-\alpha}\right)^{-1}} 2 \pi \lambda u e^{-\pi \lambda u^{2}} d u$,

where $\alpha$ is defined in (3).

Proof: See Appendix A.

B. The Result of $T_{1}^{\mathrm{NL}}$

Regarding the result of $T_{1}^{\mathrm{NL}}$, which is the coverage probability when the typical UE is associated with the typical BS with a NLoS link of distance less than $d_{1}$, we propose Lemma 3 in the following.

Lemma 3. When the typical UE is associated with a NLoS $B S$ of a distance less than $d_{1}$, the coverage probability $T_{1}^{\mathrm{NL}}$ can be computed by

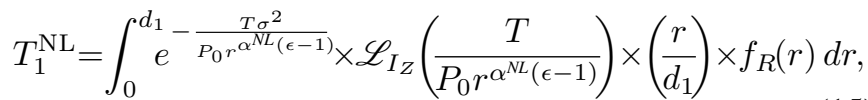

where $\mathscr{L}_{I_{Z}}\left(\frac{T}{P_{0} r^{\alpha^{N L}(\epsilon-1)}}\right)$ is the Laplace transform of $I_{Z}$ shown in (13), which is evaluated at the point $\frac{T}{P_{0} r^{\alpha^{N L}(\epsilon-1)}}$.

Proof: The proof is similar to that of Lemma 2 and is omitted for brevity.

C. The Result of $T_{2}^{\mathrm{NL}}$

Regarding the result of $T_{2}^{\mathrm{NL}}$, which is the coverage probability when the typical UE is associated with the typical BS with a NLoS link of distance greater than $d_{1}$, we propose Lemma 4 in the following.

Lemma 4. When the typical UE is associated with a NLoS BS of a distance greater than $d_{1}$, the coverage probability $T_{2}^{\mathrm{NL}}$ can be computed by

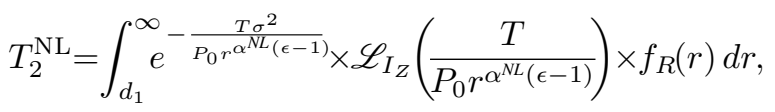

where $\mathscr{L}_{I_{Z}}\left(\frac{T}{P_{0} r^{\alpha^{N L}(\epsilon-1)}}\right)$ is the Laplace transform of $I_{Z}$ evaluated at the point $\frac{T}{P_{0} r^{\alpha^{N L}(\epsilon-1)}}$, which is expressed as

$\mathscr{L}_{I_{Z}}\left(\frac{T}{P_{0} r^{\alpha^{N L}(\epsilon-1)}}\right)=$

$\exp \left\{-2 \pi \lambda \int_{r}^{\infty}\left(\mathbb{E}_{R_{z}, \alpha^{R_{z}}}\left[\frac{1}{1+\left(P_{0} s R_{z}^{\alpha^{R_{z}} \epsilon} x^{-\alpha^{N L}}\right)^{-1}}\right]\right) x d x\right\}$,

where $\mathbb{E}_{R_{z}, \alpha}\left[\frac{1}{1+\left(P_{0} s R_{z}^{\alpha} R_{z \epsilon} x^{-\alpha^{N L}}\right)^{-1}}\right]$ is computed by (14).

Proof: The proof is similar to that of Lemma 2 and is omitted for brevity.

\section{Evaluation Using the Gauss-Laguerre Quadrature}

To improve the tractability of the derived results of (16), we propose to approximate the outer-most infinite integral in (16) by the Gauss-Laguerre quadrature [11] expressed as

$$
\int_{0}^{\infty} f(u) e^{-u} d u \approx \sum_{i=1}^{n} \omega_{i} f\left(u_{i}\right)
$$

where $n$ is the degree of Laguerre polynomial, and $u_{i}$ and $\omega_{i}$ are the $i$-th abscissas and weight of the quadrature. For practical use, $n$ should be set to a value above 10 to ensure good numerical accuracy [11].

To utilize the Gauss-Laguerre quadrature, the outer-most infinite integral in (16) is rewritten by using the change of variable $\tilde{r}=\pi \lambda r^{2}$. To evaluate (16) by means of the GaussLaguerre quadrature, we propose Lemma 5 in the following.

Lemma 5. By using the Gauss-Laguerre quadrature as shown in (18), (16) can be approximated and simplified as $T_{2}^{N L}$

$=\sum_{i=1}^{n} \omega_{i} \exp \left(-\frac{T \sigma^{2}}{P_{0}\left(\sqrt{\left[u_{i}+\pi \lambda\left(d_{1}\right)^{2}\right](\pi \lambda)^{-1}}\right)^{\alpha^{N L}(\epsilon-1)}}-\pi \lambda\left(d_{1}\right)^{2}\right)$ 


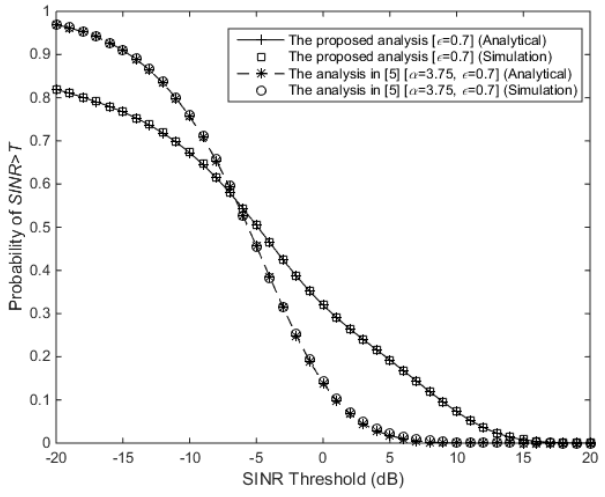

Fig. 1. The coverage probability $P^{\mathrm{cov}}(\lambda, T)$ vs. the SINR threshold with $\lambda=10 \mathrm{UEs} / \mathrm{km}^{2}$.

$$
\times \mathscr{L}_{I_{Z}}\left(\frac{T}{P_{0} \sqrt{\left[u_{i}+\pi \lambda\left(d_{1}\right)^{2}\right](\pi \lambda)^{-1}} \alpha^{N L}(\epsilon-1)}\right) .
$$

Proof: See Appendix B.

Thanks to Lemma 5, the 3-fold integral computation in (16) can now be simplified as a 2 -fold integral computation, which improves the tractability of our results.

\section{Simulation And Discussion}

In this section, we present numerical results to establish the accuracy of our analysis and further study the performance of the UL of dense SCNs. We adopt the following parameters according to the 3GPP recommendations [13]: $d_{1}=0.3 \mathrm{~km}$, $\alpha^{\mathrm{L}}=2.09, \alpha^{\mathrm{NL}}=3.75, P_{0}=-76 \mathrm{dBm}, \sigma^{2}=-99 \mathrm{dBm}$ (with a noise figure of $5 \mathrm{~dB}$ at each $\mathrm{BS}$ ). We first consider a sparse network in subsections IV-A and IV-B, and then we analyze a dense network in the subsections IV-C and IV-D.

\section{A. Validation of the Analytical Results of $P^{\mathrm{cov}}(\lambda, T)$}

The results of $P^{\text {cov }}(\lambda, T)$ in a sparse deployment with $\lambda=10 \mathrm{UEs} / \mathrm{km}^{2}$ are plotted in Fig. 1. For comparison, we have also included analytical results using a single-slope path loss model that does not differentiate LoS and NLoS transmissions [5]. Note that in [5], only one path loss exponent is defined and denoted by $\alpha$, the value of which is $\alpha=\alpha^{\mathrm{NL}}=3.75$. As can be observed from Fig. 1, our analytical results perfectly match the simulation results, and thus we will only use analytical results of $P^{\text {cov }}(\lambda, T)$ in our discussion hereafter.

\section{B. The Results of $P^{\mathrm{cov}}(\lambda, T)$ with Various $\epsilon$}

The results of $P^{\text {cov }}(\lambda, T)$ in a sparse deployment with $\lambda=10 \mathrm{UEs} / \mathrm{km}^{2}$ for various $\epsilon$ are plotted in Fig. 2. From Fig. 2, we can observe that the coverage probability performance improves as $\epsilon$ increases. This is because sparse cellular networks generally work in the noise-limited region, and thus

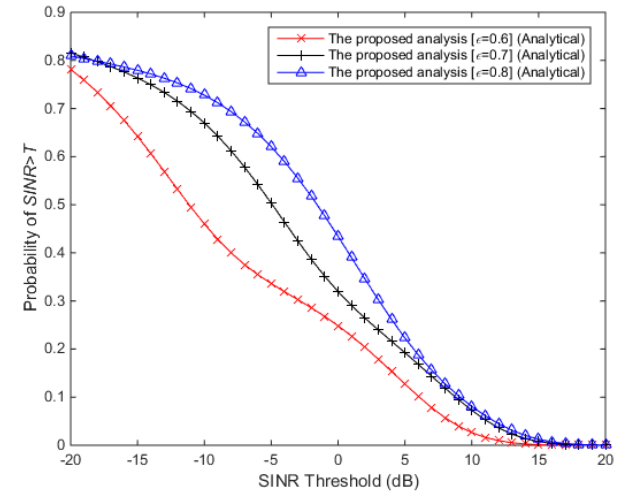

Fig. 2. The coverage probability $P^{\operatorname{cov}}(\lambda, T)$ vs. the SINR threshold with various power compensation factor $\epsilon\left(\lambda=10 \mathrm{UEs} / \mathrm{km}^{2}\right)$.

a larger $\epsilon$ allows more transmission power to combat the link noise and achieve better performance. However, it is of great interest to see whether such observation still holds for other network scenarios, especially the dense SCNs.

\section{The Results of $P^{\mathrm{cov}}(\lambda, T)$ vs. $\lambda$}

The results of $P^{\text {cov }}(\lambda, T)$ with the UE density for $T=0 \mathrm{~dB}$ are plotted in Fig. 3. From Fig. 3, we can observe that when there is no LoS/NLoS modeling [5], the coverage probability first increases with the UE density at a fast pace because a smaller UE-to-BS distance results in better coverage in noiselimited networks. Thereafter, the increase of the coverage probability slows down when $\lambda$ is large enough, since the network is pushed into the interference-limited region. In contrast, when considering both LoS and NLoS transmissions, the coverage probability presents a significantly different behaviour. When the SCN is sparse and thus noise-limited, the coverage probability given by the proposed analysis grows as $\lambda$ increases, similarly as in [5]. However, when the network is dense enough, the coverage probability decreases as $\lambda$ increases, due to the transition of a large number of interference paths from NLoS to LoS, which is not captured in [5]. In more detail, the coverage probability given by the proposed analysis peaks at $\lambda_{0}$, and when $\lambda$ increases above $\lambda_{0}$, interfering UEs are so close to the typical BS that their interfering signals start reaching it via strong LoS paths too. When $\lambda$ is further increased far above $\lambda_{0}$, the coverage probability decreases at a slower pace because both the signal power and the interference power are LoS dominated and thus statistically stable.

It should also be noted that the coverage probability with different $\epsilon$ has different trends. Specifically, when the SCN is sparse, adopting a higher $\epsilon$ (e.g., $\epsilon=0.8$ ) leads to higher coverage probability. This is because the SCN is noise-limited and hence increasing the transmission power provides better coverage. However, as $\lambda$ increases, adopting a lower $\epsilon$ (e.g., $\epsilon=0.6)$ leads to higher coverage probability. Therefore, our results suggest that in dense SCNs, increasing the UL transmission power may degrade the coverage probability. 


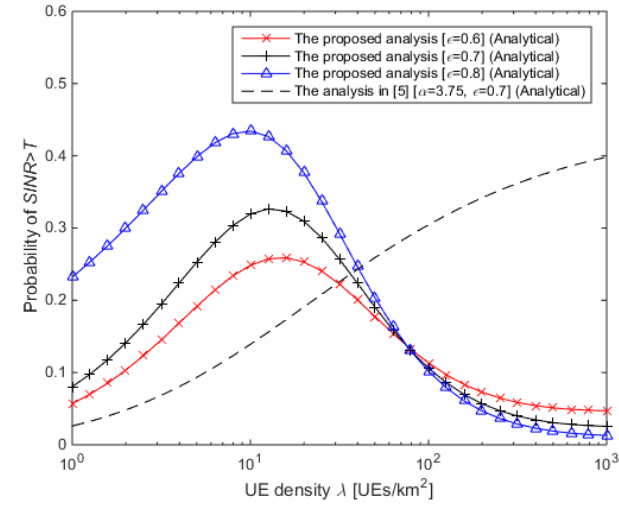

Fig. 3. The coverage probability $P^{\mathrm{cov}}(\lambda, T)$ vs. the UE density with different $\epsilon$ and SINR threshold $T=0 \mathrm{~dB}$

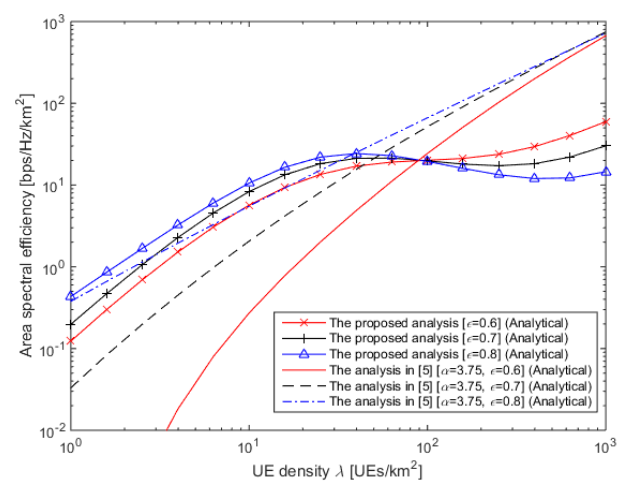

Fig. 4. Area spectral efficiency $A^{\mathrm{ASE}}\left(\lambda, T_{0}\right)$ vs. the UE density with different $\epsilon$ and SINR threshold $T_{0}=0 \mathrm{~dB}$.

Such observation is further investigated in terms of ASE in the following subsection.

\section{The Results of $A^{\mathrm{ASE}}\left(\lambda, T_{0}\right)$ vs. $\lambda$}

In this subsection, we investigate the analytical results of $A^{\mathrm{ASE}}\left(\lambda, T_{0}\right)$ with $T_{0}=0 \mathrm{~dB}$ based on the analytical results of $P^{\operatorname{cov}}(\lambda, T)$. The results of $A^{\mathrm{ASE}}\left(\lambda, T_{0}\right)$ obtained by comparing the proposed analysis with the analysis from [5] are plotted in Fig. 4.

As can be seen from Fig. 4, the analysis from [5] indicates that when the SCN is dense enough, the ASE increases linearly with $\lambda$. In contrast, our proposed analysis reveals a more complicated ASE trend. Specifically, when the SCN is relatively sparse, i.e., $10^{0} \sim 10^{1} \mathrm{UEs} / \mathrm{km}^{2}$, the ASE quickly increases with $\lambda$ since the network is generally noise-limited, and thus having UEs closer to their serving BSs improves performance. When the SCN is extremely dense, i.e., around $10^{3} \mathrm{UEs} / \mathrm{km}^{2}$, the ASE increases linearly with $\lambda$ because both the signal power and the interference power are LoS dominated and thus statistically stable as explained before. As for the practical range of $\lambda$ for the existing and the future cellular networks, i.e., $10^{1} \sim 10^{3} \mathrm{UEs} / \mathrm{km}^{2}$ [1], the ASE trend is interesting. First, when $\lambda \in\left[\lambda_{0}, \lambda_{1}\right]$, where $\lambda_{0}$ is around 20 and $\lambda_{1}\left(\lambda_{1}>\lambda_{0}\right)$ is around 100 in Fig. 4, the ASE exhibits a slowing-down in the rate of growth $(\epsilon=0.6)$ or even a decrease $(\epsilon=0.7,0.8)$ due to the fast decrease of coverage probability shown in Fig. 3. Thereafter, when $\lambda \geq \lambda_{1}$, the ASE exhibits an acceleration in the growth rate due to the slow-down in the decrease of coverage probability also shown in Fig. 3. Our finding, the ASE may decrease as the UE density increases, is similar to our results reported for the DL of SCNs [9], which indicates the significant impact of the path loss model incorporating both NLoS and LoS transmissions. Such impact makes a difference for dense SCNs in terms of the ASE both quantitatively and qualitatively, comparing to that with a simplistic path loss model that does not differentiate LoS and NLoS transmissions.

Our proposed analysis also shows another important finding. A smaller UL power compensation factor $\epsilon$ (e.g., $\epsilon=0.6$ ) can greatly boost the ASE performance in $5 \mathrm{G}$ dense SCNs [1], i.e., $10^{2} \sim 10^{3} \mathrm{UEs} / \mathrm{km}^{2}$, while a larger $\epsilon$ (e.g., $\left.\epsilon=0.8\right)$ is more suitable for sparse SCNs, i.e., $10^{0} \sim 10^{2} \mathrm{UEs} / \mathrm{km}^{2}$. This contradicts the results in [5] where a larger UL power compensation factor was predicted to always result in a better ASE in the practical range of UE density, i.e., $10^{0} \sim 10^{3} \mathrm{UEs} / \mathrm{km}^{2}$, as shown in Fig. 4. Therefore, our theoretical analysis indicates that the performance impact of LoS and NLoS transmissions on UL SCNs with UL power compensation is also significant both quantitatively and qualitatively, compared with the previous work in [5] that does not differentiate among those. Interestingly, our new finding implies that its is possible to save UE battery and meanwhile achieve high ASE in the UL of $5 \mathrm{G}$ dense SCNs, if $\epsilon$ is optimized. The intuition is that in dense SCNs, the network experiences a surplus of strong LoS interference instead of shortage of UL transmission power, and thus decreasing the transmission powers of a large number of interferers turns out to be a good strategy that enhances the ASE. Note that our conclusion is made from the investigated set of parameters, and it is of significant interest to further study the generality of this conclusion in other network models and with other parameter sets.

\section{CONCLUSiON}

In this paper, we have investigated the impact of a piecewise linear path loss model incorporating both LoS and NLoS transmissions in the performance of the UL of dense SCNs. Analytical results were obtained for the coverage probability and the ASE performance. The results show that LoS and NLoS transmissions have a significant impact in the ASE of the UL of dense SCNs, both quantitatively and qualitatively, compared with previous works that does not differentiate LoS and NLoS transmissions. Specifically, we found that

- The ASE may suffer from a slow growth or even a decrease as the UE density increases.

- The ASE with a smaller UL power compensation factor considerably outperforms that with a larger UL power compensation factor in dense SCNs. The other way around is true for sparse SCNs. 
As our future work, we will consider more practical assumptions, such as an alternative UE association strategy (UAS), where each UE is connected to the BS that gives the smallest pathloss. Note that such UAS will blur the boundaries of UEs' Voronoi cells, because a UE is no longer always connected to its closest BS.

\section{ACKNOWLEDGEMENT}

This research is supported by Australian Research Council (ARC) Discovery projects DP110100538 and DP120102030 and Chinese National Science Foundation project 61428102.

\section{Appendix A: PRoOf of Lemma 2}

According to system model addressed in Section II, $T_{1}^{\mathrm{L}}$ can be derived as

$T_{1}^{\mathrm{L}}$

$=\int_{0}^{d_{1}} \operatorname{Pr}\left(\frac{P g r^{\alpha^{\mathrm{L}}(\epsilon-1)}}{\sigma^{2}+I_{Z}}>T \mid \operatorname{LoS}\right) \times\left(1-\frac{r}{d_{1}}\right) \times f_{R}(r) d r$

$=\int_{0}^{d_{1}} \operatorname{Pr}\left(g>\frac{T\left(\sigma^{2}+I_{Z}\right)}{\operatorname{Pr}^{\alpha^{L}(\epsilon-1)}}\right) \times\left(1-\frac{r}{d_{1}}\right) \times f_{R}(r) d r$

$=\int_{0}^{d_{1}} \mathbb{E}_{I_{Z}}\left[\exp \left(-\frac{T\left(\sigma^{2}+I_{Z}\right)}{P r^{\alpha^{L}(\epsilon-1)}}\right)\right] \times\left(1-\frac{r}{d_{1}}\right) \times f_{R}(r) d r$

$=\int_{0}^{d_{1}} e^{-\frac{T \sigma^{2}}{P r^{\alpha^{L}}(\epsilon-1)}} \times \mathbb{E}_{I_{Z}}\left[\exp \left(-\frac{T I_{Z}}{P r^{\alpha^{L}}(\epsilon-1)}\right)\right]$

$\times\left(1-\frac{r}{d_{1}}\right) \times f_{R}(r) d r$

$=\int_{0}^{d_{1}} e^{-\frac{T \sigma^{2}}{P r^{\alpha}(\epsilon-1)}} \times \mathscr{L}_{I_{Z}}\left(\frac{T}{P r^{\alpha^{L}(\epsilon-1)}}\right) \times\left(1-\frac{r}{d_{1}}\right) \times f_{R}(r) d r$.

The Laplace transform $\mathscr{L}_{I_{Z}}(s)$ can be derived as

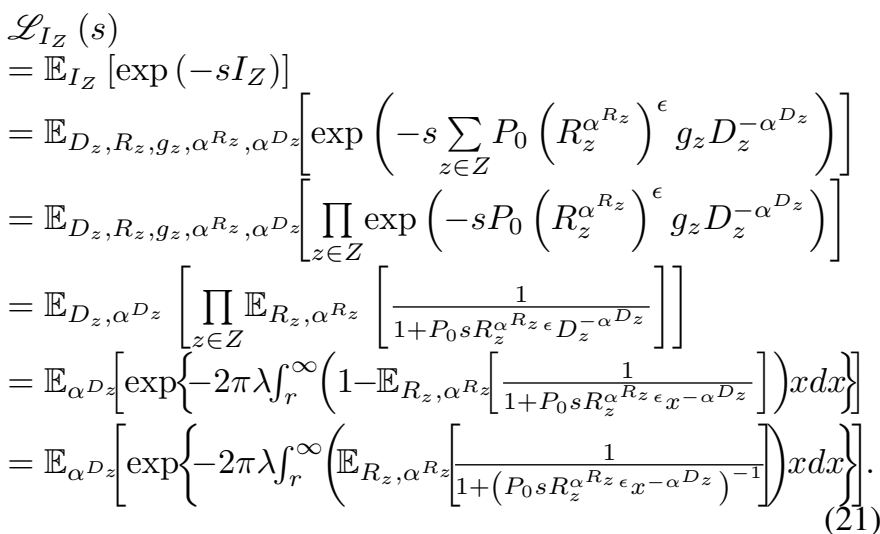

The locations of interferers can be divided according to the distance into LoS and NLoS path sets. So the $\mathscr{L}_{I_{Z}}(s)$ can be further derived as shown in (13).

The average function in the Laplace transform can be derived as

$$
\begin{aligned}
& \mathbb{E}_{R_{z}, \alpha R_{z}}\left[\frac{1}{1+\left(P_{0} s R_{z}^{\alpha^{R}} \epsilon_{x^{-\alpha}}\right)^{-1}}\right] \\
& =\mathbb{E}_{\alpha^{R_{z}}}\left[\int_{0}^{\infty} \frac{1}{1+\left(P_{0} s u^{\alpha^{u}} x^{-\alpha}\right)^{-1}} 2 \pi \lambda u e^{-\pi \lambda u^{2}} d u\right] \\
& =\int_{0}^{d_{1}}\left(1-\frac{u}{d_{1}}\right) \frac{1}{1+\left(P_{0} s u^{\alpha^{L}} \epsilon x^{-\alpha}\right)^{-1}} 2 \pi \lambda u e^{-\pi \lambda u^{2}} d u \\
& \quad+\int_{0}^{d_{1}} \frac{u}{d_{1}} \frac{1}{1+\left(P_{0} s u^{\alpha^{N L}} \epsilon^{-\alpha}\right)^{-1}} 2 \pi \lambda u e^{-\pi \lambda u^{2}} d u \\
& =\int_{0}^{d_{1}}\left(1-\frac{u}{d_{1}}\right) \frac{1}{1+\left(P_{0} s u^{\alpha^{L}} \epsilon_{x^{-\alpha}}\right)^{-1}} 2 \pi \lambda u e^{-\pi \lambda u^{2}} d u
\end{aligned}
$$

$$
\begin{aligned}
& +\int_{0}^{d_{1}} \frac{u}{d_{1}} \frac{1}{1+\left(P_{0} s u^{\alpha^{N} \epsilon_{\epsilon}} x^{-\alpha}\right)^{-1}} 2 \pi \lambda u e^{-\pi \lambda u^{2}} d u \\
& +\int_{d_{1}}^{\infty} \frac{1}{1+\left(P_{0} s u^{\alpha^{N} L_{\epsilon}} x^{-\alpha}\right)^{-1}} 2 \pi \lambda u e^{-\pi \lambda u^{2}} d u .
\end{aligned}
$$

\section{APPENDIX B: PROOF OF LEMMA 5}

By using the change of variable $\pi \lambda r^{2} \rightarrow \tilde{r}$, (16) can be rewritten as

$$
\begin{aligned}
T_{2}^{\mathrm{NL}} & =\int_{\pi \lambda d_{1}^{2}}^{\infty} \exp \left(-\frac{T \sigma^{2}}{P_{0}\left(\sqrt{\widetilde{r}(\pi \lambda)^{-1}}\right)^{\alpha^{\mathrm{NL}}(\epsilon-1)}}\right) \\
& \times \mathscr{L}_{I_{Z}}\left(\frac{T}{P_{0}\left(\sqrt{\widetilde{r}(\pi \lambda)^{-1}}\right)^{\alpha^{\mathrm{NL}}(\epsilon-1)}}\right) \times e^{-\widetilde{r}} d \widetilde{r} .
\end{aligned}
$$

By using the change of variable $\widetilde{r}-\pi \lambda\left(d_{1}\right)^{2} \rightarrow v$, (23) can be rewritten as

$$
\begin{aligned}
& T_{2}^{\mathrm{NL}}=\int_{0}^{\infty} \exp \left(-\frac{T \sigma^{2}}{P_{0}\left(\sqrt{\left[v+\pi \lambda\left(d_{1}\right)^{2}\right](\pi \lambda)^{-1}}\right)^{\alpha^{\mathrm{NL}}(\epsilon-1)}}\right) \\
& \times \mathscr{L}_{I_{Z}}\left(\frac{T}{P_{0}\left(\sqrt{\left[v+\pi \lambda\left(d_{1}\right)^{2}\right](\pi \lambda)^{-1}}\right)^{\alpha^{\mathrm{NL}}(\epsilon-1)}}\right) \times e^{-\pi \lambda\left(d_{1}\right)^{2}} \times e^{-v} d v .
\end{aligned}
$$

By using the method of Gauss-Laguerre quadrature as shown in (18), our proof is finished.

\section{REFERENCES}

[1] D. López Pérez, M. Ding, H. Claussen, and A. H. Jafari, "Towards $1 \mathrm{Gbps} / \mathrm{UE}$ in cellular systems: Understanding ultra-dense small cell deployments," IEEE Commun. Surveys and Tutorials, Jun. 2015. (Available: http://arxiv.org/abs/1503.03912)

[2] X. Ge, S. Tu, G. Mao, C.-X. Wang and T. Han, "5G Ultra-Dense Cellular Networks," IEEE Wireless Communications, vol. 23, no. 1, Feb. 2016.

[3] 3GPP, "TR 36.872, Small cell enhancements for E-UTRA and EUTRAN - Physical layer aspects," Dec. 2013.

[4] J. G. Andrews, F. Baccelli, and R. K. Ganti, "A tractable approach to coverage and rate in cellular networks," IEEE Trans. on Commun., vol. 59, no. 11, pp. 3122-3134, Nov. 2011.

[5] T. D. Novlan, H. S. Dhillon, and J. G. Andrews, "Analytical modeling of uplink cellular networks," IEEE Trans. on Wireless Commun., vol. 12, no. 6, pp. 2669-2679, Jun. 2013

[6] Y. Hu, Y. Hong, and J. Evans, "Uplink Coverage and Spatial Blocking in Poisson Cellular Networks," IEEE ICC 2014, Sydney, Australia, pp. 5765 - 5770, Jun. 2014

[7] S. Singh, X. Zhang, and J. G. Andrews, "Uplink Rate Distribution in Heterogeneous Cellular Networks with Power Control and Load Balancing," IEEE ICC Workshop 2015, London, UK, pp. 1275 - 1280, Jun. 2015.

[8] H. ElSawy, and E. Hossain, "On Stochastic Geometry Modeling of Cellular Uplink Transmission With Truncated Channel Inversion Power Control," IEEE Trans. on Wireless Commun., vol. 13, no. 8, pp. 44544469, Aug. 2014.

[9] M. Ding, P. Wang, D. López-Pérez, G. Mao, and Z. Lin, "Performance Impact of LoS and NLoS Transmissions in Small Cell Networks", submitted to IEEE Trans. on Wireless Commun., arXiv:1503.04251 [cs.IT], Mar. 2015.

[10] Spatial Channel Model AHG (Combined ad-hoc from 3GPP \& 3GPP2), "Subsection 3.5.3, Spatial Channel Model Text Description V6.0," Apr. 2003.

[11] C. Pozrikidis, "Numerical computation in science and engineering," New York: Oxford university press, 1998.

[12] 3GPP, "TS 36.213 (V11.2.0): Physical layer procedures," Feb. 2013.

[13] 3GPP, "TR 36.828 (V11.0.0): Further enhancements to LTE Time Division Duplex (TDD) for Downlink-Uplink (DL-UL) interference management and traffic adaptation," Jun. 2012. 